Review Article

\title{
Covid-19 and Pregnancy Evidence Review
}

\author{
Antonio Ulises Cusihuamán Puma,* Julia Raquel Medina Rodríguez \\ Ministry of Public Health and Welfare, Directorate of Sexual and Reproductive Health, Paraguay
}

\begin{abstract}
Introduction: The Covid-19 pandemic has raised concern about the impact on pregnancy and the possibility of vertical mother-child transmission of SARS-CoV-2.

Method: The present study aimed to review published literature regarding the implications of Covid-19 in pregnancy, from December 2019 to the present date, collected from various databases, applying the MeSH descriptor for keywords compatible with SARS-CoV-2, Covid-19, pregnancy and vertical transmission, precisely, and the Zotero bibliographic reference manager in the selected articles. The description of the findings of this study were organized into four topics: clinical presentation and evolution in pregnant women with Covid-19, universal test for SARS-CoV-2 and evaluation of vertical transmission, management of pregnant women with Covid-19 in the health system, breastfeeding in the context of Covid-19 and risk of contagion to the newborn.
\end{abstract}

Developing and Discussion: Although most of the clinical symptoms of Covid-19 in pregnant women are not different from non-pregnant women, the obstetric population has required and shown a higher risk of admission to the Intensive Care Unit and mechanical ventilation than non-pregnant women. Vertical transmission of SARS-CoV-2 in pregnancy is not ruled out.

Conclusion: More evidence is required about the effect of SARS-CoV-2 on pregnancy. In accordance with a management safety protocol established in the health system network, it will be necessary to monitor thoroughly pregnant women considered suspected or confirmed cases of Covid-19 before and after delivery, as well as their children in both the fetal and neonatal periods.

Keywords: Health contingency Covid-19, Pregnancy, Vertical transmission, SARS-CoV-2, Paraguay (MeSH).

\section{Introduction}

Vertical transmission refers to the passage of a pathogen from mother to child during the perinatal period, that is, before and after birth. Specifically, it includes transmission through germ cells, the placental hematogenous route during pregnancy, through the birth canal during delivery, and during postpartum lactation. ${ }^{1}$ Intrauterine vertical transmission is one of the most serious complications of viral diseases during pregnancy. It can occur with maternal infection by congenitally transmitted TORCH agents (acronym for Toxoplasma, Others, Rubella, Cytomegalovirus, Herpes), and recently, they also include Zika and Ebola viruses. Maternal-fetal transmission of viral diseases (with the exception of the herpes virus) is usually through the hematogenous route, in which the virus that circulates through the maternal bloodstream enters the placenta, reaching the chorionic villous tree and the vessels fetal blood, and is transmitted to the fetus. ${ }^{2}$

\section{Epidemiology of the new coronavirus}

In December 2019, the World Health Organization (WHO) warned of several cases of pneumonia detected in the Chinese city of Wuhan caused by an unknown virus. ${ }^{3}$ In January 2020, the Chinese authorities confirmed the identification of a new coronavirus that currently represents a public health problem worldwide, from the same family as other viruses that cause respiratory infections, called severe acute respiratory syndrome coronavirus 2 (SARSCoV-2) or 2019-nCoV, the seventh member of the coronavirus family that infects humans (HCoV), ${ }^{4}$ which produces Covid-19, a disease

\begin{tabular}{|l|l|}
\hline Quick Response Code: & *Corresponding author: Antonio Ulises Cusihuamán Puma, Ministry of Public Health and Wel- \\
fare, General Directorate of Programs, Directorate of Sexual and Reproductive Health, Asunción, \\
Paraguay \\
Received: 23 April, 2021 \\
Citation: Cusihuamán-Puma AU, Medina-Rodríguez JR. Covid-19 and Pregnancy Evidence Re- \\
view. Pregn Womens Health Care Int J. 2021;1(2):1-9. DOI: 10.53902/PWHCIJ.2021.01.000507
\end{tabular}


that can range from mild to moderate, severe respiratory conditions to potentially critical conditions fatalities with multiple organ failure due to an inflammatory and/or thrombotic storm..$^{5-7}$ And that to date, more than 140 million cases have been reported in the world since the beginning of the pandemic, of which 18.5 million $(12.9 \%)$ are currently active $(99.4 \%$ with mild to moderate symptoms, and $0.6 \%$ in critical condition) and more than 3 million deaths, ${ }^{8}$ which reflects an overall fatality rate of $2.1 \%$.

It is important to note that the other two coronavirus infections that were reported years ago and with serious adverse results in pregnancy: severe acute respiratory syndrome (SARS) caused by SARS-CoV in 2003 and the Middle East respiratory syndrome (MERS) caused by MERS-CoV in 2012, ${ }^{4}$ included maternal morbidity and mortality, maternal-fetal transmission of the virus and perinatal infections, spontaneous abortion in the first trimester, intrauterine growth restriction in the second trimester, preterm delivery. 2,9 Then we have other HCoV infections including HCoV 229E, NL63, OC43, and HKU1, which generally cause mild to moderate upper respiratory illness such as the common cold. ${ }^{10}$ Although Covid-19 appears as a disease that primarily affects the lung areas, it has also been identified in neurological, digestive, vascular and skin areas. ${ }^{6,7}$

\section{Importance of prenatal control}

Maternity care is essential and has been developed over many years to reduce maternal and child complications. The risks of not attending prenatal care include harm to both the mother, the fetus or both, even in the context of Covid-19. Since the gestation process in women involves physiological changes that do not exempt them from contracting respiratory viral infections, pregnant women with respiratory diseases must receive priority treatment, as they have a greater risk of suffering adverse effects. ${ }^{11}$ Remember that a first prenatal control is recommended before 12 weeks of gestation. And also, pregnant women are always advised to receive a flu vaccination (influenza vaccine) if posible. ${ }^{12}$ The current spread of the new coronavirus (SARS-CoV-2) generates a social alert in the world, especially to the group of pregnant women regarding the effects of this disease on them and their future babies, ${ }^{2}$ raising the following questions:

Are the symptoms of Covid-19 infection different in pregnant women and non-pregnant women?

\section{Is vertical maternal-fetal or neonatal transmission possible?}

What measures should be taken for the health of the mother-child binomial?

The objective of this article was to review the published literature regarding the clinical characteristics of Covid-19 in pregnancy, the potential vertical transmission and the pertinent recommendations.

\section{Methodology}

The present study is a descriptive, observational, cross-sectional and retrospective review of the compiled literature regard- ing Covid-19 in pregnancy. To do this, the articles published since December 2019 to date in various databases were accessed, to mention, Elsevier, Embase, Medline, NEJM, PubMed, SciELO, Scopus, Google and Google Scholar, with a personal subscription to Researchgate, applying the following phases:

1) Identification of relevant publications

2) Sequential application of keywords compatible with the MeSH descriptor and its corresponding name in Spanish, including Covid-19, pregnancy, vertical transmission, Coronavirus 2019, SARS-CoV-2 or 2019- nCoV

3) For the definition of the selected articles, the titles, abstracts and extensive publications were reviewed

4) The Zotero bibliographic reference manager was used.

There were collected all the original research, case studies or case series, articles published in form of correspondence, commentary or editorial, and some press releases and health area management guides that addressed the subject, so the scientific literature in English and Spanish was selected, excluding articles written purely in Chinese, duplication or animal research and those that were not articles.

The different research studies accessed, indistinctly, the natural history of the disease during the perinatal period of pregnant women with Covid-19 infection, including demographic, epidemiological data, clinical records such as symptoms, laboratory results, radiological and computed tomography (CT) images of the chest, treatment and the state of labor and the puerperium, screening for SARS-CoV-2 in pregnant women using polymerase chain reaction-transcription (rt-PCR) of nasopharyngeal swab or oropharyngeal $^{2,7}$ and/or suggestive findings on chest CT. ${ }^{13-16}$ The information of the newborns of each mother with Covid-19, included clinical symptoms, laboratory reports, chest X-ray images and treatment. Evidence of vertical transmission was evaluated by testing for the presence of SARS-CoV-2 in samples of amniotic fluid, umbilical cord blood, placenta, genital fluid, and neonatal oropharyngeal swab in real time using rt-PCR. ${ }^{17}$ Breast milk samples (colostrum) were also collected. ${ }^{10,18}$ And some histopathological studies of the placenta were carried out. ${ }^{19,20}$ Other articles were about informative nature, concerning guidelines and recommendations based on the evidence of the moment.

The findings were organized into four themes: clinical presentation and evolution of pregnant women with Covid-19; universal test for SARS-CoV-2 and evaluation of vertical transmission; management of pregnant women with Covid-19 in the health system; breastfeeding and risk of contagion to the newborn within the context of Covid-19.

\section{Development and Discussion}

Pregnant women with Covid-19, clinical picture and evolution: On the one hand, a multicenter study was carried out that included 13,118 pregnant women (14\%) and 83,486 non-pregnant women 
(86\%) with Covid-19, respectively, between December 1 from 2019 to June 26, 2020, covering 77 cohort studies conducted: 26 from the United States, 24 from China, seven from Italy, six from Spain, three from both the United Kingdom and France, and one from Belgium, Brazil, Denmark, Israel, Japan, Mexico, the Netherlands and Portugal, respectively. ${ }^{15}$ And another meta-analysis that reported 326,335 women of childbearing age (WCBA) with positive results for SARS-CoV-2 in the USA that among them, 8,207 were pregnant, representing $2.5 \%$ of all WCBA with Covid-19 between January 22 and June 7, 202016 (Figure 1).

All studies analyzed respiratory samples using rt- PCR to confirm the presence of SARS-CoV-2, although initially other studies were based on clinical suspicion and single chest CT. ${ }^{13,14}$ Among
WCBAs aged 15 to 44 , approximately $25 \%$ of the women were between 15 and 24 years old. 54\% of pregnant women and $38 \%$ of non-pregnant women were between 25 and 34 years old; $22 \%$ of the pregnant women and $38 \%$ of the non-pregnant women were between 35 and 44 years old ${ }^{16}$ (Table 1 ).

Then, the frequency of the most common symptoms reported by pregnant and newly pregnant women with suspected or confirmed Covid-19 were: fever 40\% (95\% CI:22-85), cough 39\% (95\% CI:29-49) and myalgia 48\% (95\% CI:45-51); lymphopenia 35\% (95\% CI:26-45) and elevated levels of C-reactive protein 49\% (95\% CI:39-59), as the most common laboratory findings ${ }^{15}$ (Figure 2).

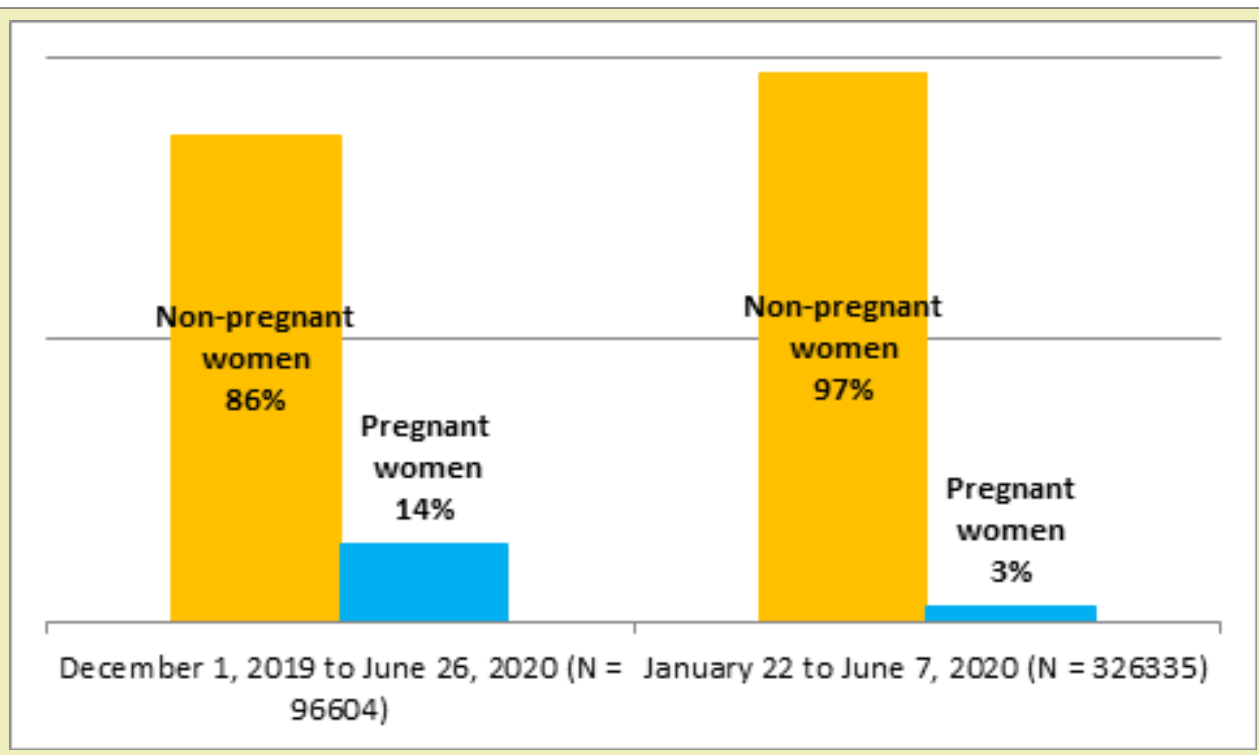

Figure 1: Number of women diagnosed with Covid-19

Source: Clinical manifestations, risk factors, and maternal and perinatal outcomes of coronavirus disease 2019 in pregnancy: living systematic review and meta-analysis. BMJ. September 1, 2020 (N=96604) ${ }^{15}$ Characteristics of Women of Reproductive Age with Laboratory-Confirmed SARS-CoV-2 Infection by Pregnancy Status-United States. MMWR. Morbidity and Mortality Weekly Report. June 26, 2020 (N=326335) ${ }^{16}$

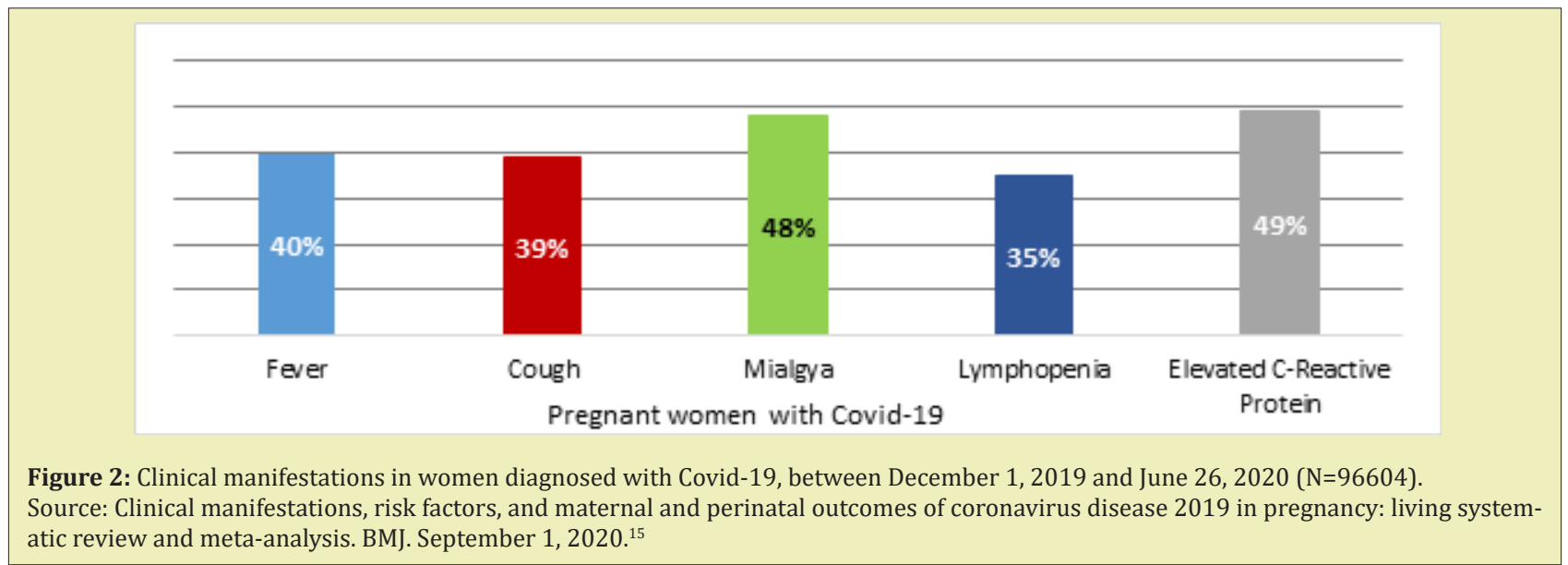


Table 1: Women diagnosed with Covid-19, according to age group, between January 22 and June 7, $2020(\mathrm{~N}=326335)$.

\begin{tabular}{|l|l|l|}
\hline \multicolumn{3}{|l|}{ Age } \\
\hline 15 - 24 years WCBA & $25 \%$ & IC95\% 17-35 \\
\hline 25 - 34 years Pregnant women & $54 \%$ & IC95\% 44-64 \\
\hline 25 - 34 years Non-pregnant women & $38 \%$ & IC95\% 29-48 \\
\hline 35 - 44 years Pregnant women & $22 \%$ & IC95\% 14-31 \\
\hline 35 - 44 years Non-pregnant women & $38 \%$ & IC95\% 29-48 \\
\hline
\end{tabular}

Although most of the clinical symptoms of Covid-19 in pregnant women are not different from non-pregnant women (mild symptoms); However, both meta-analyzes show that the obstetric population required hospital admission 5.4 times more (95\% CI:5.1-5.6) than non-pregnant women, although there were no data available to distinguish hospitalization due to circumstances related to Covid-19, and 1.5 times more risk of entering the Intensive Care Unit (ICU) (95\% CI:1.2-1.8) and 1.7 more times of requiring mechanical ventilation (95\% CI:1.2-2.4) (serious and critical pictures). No differences were found in the risk of death between pregnant and non-pregnant women 0.9 (95\% CI:0.5-1.5). It should be mentioned that in these studies all outcomes (hospitalization, ICU ad- mission, receipt of mechanical ventilation, and death) were reported more frequently among women aged 35 to 44 years than among those aged 15 to 24, regardless of pregnancy status Older maternal age 2\% (95\% CI:1-3). Later, underlying chronic conditions were reported for both pregnant and non-pregnant women ${ }^{16}$ (Figure 3). In addition, it was identified: high body mass index 24\% (95\% CI:1734), chronic arterial hypertension 20\%( 95\% CI:11.4-34.8). ${ }^{15}$

On the other hand, in a multicenter study and case report (from Australia, Canada, China, Korea, USA, Spain, France, India, Ireland, Iran, Italy, Jordan, Netherlands, Peru, United Kingdom, Sweden and Turkey) which reported 790 women with positive Covid-19 and 548 newborns, between December 2019 to July 8, 2020, the rates of caesarean section, preterm delivery, low birth weight and adverse pregnancy events were estimated at $72 \%, 23 \%, 7 \%$ and $27 \%$, respectively. In the analysis of heterogeneity, the caesarean section rate was substantially higher in the Chinese (91\%) studies compared to the US (40\%) or European (38\%) studies. Rates of preterm birth and adverse pregnancy events were also lower in the US $(12 \%, 15 \%)$ studies compared to the Chinese $(17 \%, 21 \%)$ and European $(19 \%, 19 \%)$ studies) $)^{21}$ (Figure 4).

Case reports from Korea, India, the Netherlands, Australia, Italy,

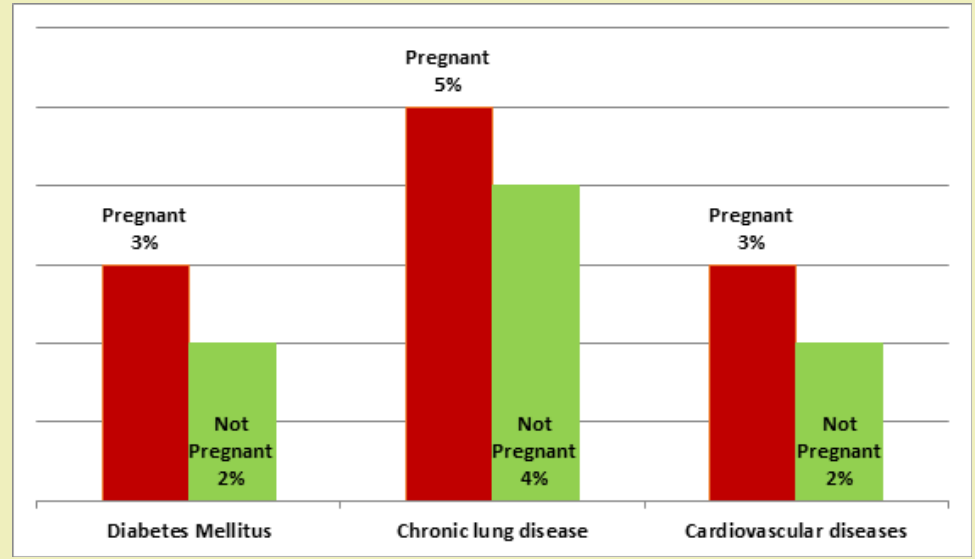

Figure 3: Chronic conditions reported more frequently in women diagnosed with Covid-19, between January 22 to June 7, 2020 (N=326335). Source: Characteristics of Women of Reproductive Age with Laboratory -Confirmed SARS-CoV-2 Infection by Pregnancy Status - United States. MMWR. Morbidity and Mortality Weekly Report. June 26, 2020.16

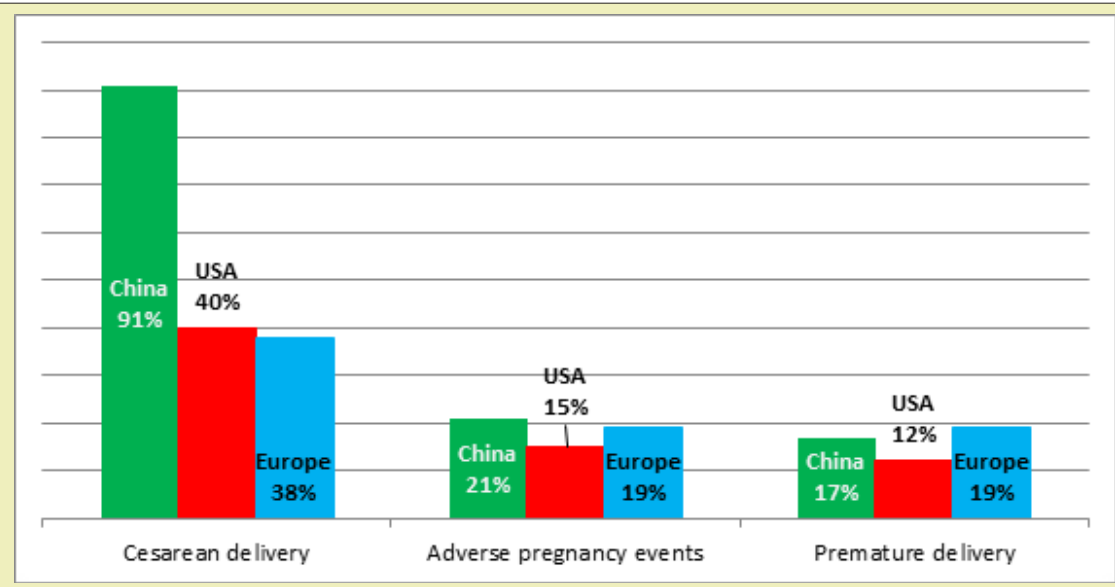

Figure 4: Evolution of pregnancy according to geographic regions in women diagnosed with Covid-19, between December 2019 and July 8 , $2020(\mathrm{~N}=790, \mathrm{n}=548)$. Source: Maternal and neonatal characteristics and outcomes among COVID-19 infected women: An updated systematic review and meta-analysis. ${ }^{21}$ 
Ireland, and Turkey did not show any adverse pregnancy outcomes. All of these cases were detected after the gestation age of 35 weeks except for one case in one study. However, cases from Peru, Spain, Iran, and the United Kingdom showed adverse pregnancy outcomes that may be due to early detection of infection before the gestational age of 35 weeks. ${ }^{21}$ In other previous, smaller-scale studies, the symptoms of pregnant women with Covid-19 similar to nonpregnant ones had already been described, mainly with fever and cough, and eventually diarrhea ${ }^{22}$ in mild to moderate conditions; and in severe cases: sore throat, dyspnea and fatigue, with the respiratory symptoms of Covid-19 being predominant. ${ }^{16,23}$ It should be noted that within a cross-sectional study, it is described that women, some of them pregnant, manifested anosmia and ageusia more frequently than men as initial symptoms of Covid-19, later adding respiratory symptoms $(10 / 19[52.6 \%]$ vs $10 / 40[25 \%]$; $\mathrm{P}=.036) .{ }^{24}$ Then we describe cases of pregnant patients who presented deterioration during hospitalization, which led to admission to the ICU with multiple organ dysfunction syndrome (MODS) that required intubation and ventilatory mechanics, acute liver failure, acute kidney failure and septic shock, requiring, in addition, ECMO support., ${ }^{7,25}$ Evaluation of vaginal or cesarean delivery in mothers with Covid-19 showed that neither type of delivery affected their newborns and all neonates studied were negative for Covid-19 infection. ${ }^{17,18}$ With respect to the neonates, the majority had a favorable evolution with a good Apgar score. In the case of premature infants, they presented with respiratory distress, fever, impaired liver function, tachycardia, vomiting or pneumothorax, and some death. Neonatal asphyxia was not observed. ${ }^{17,18}$ Transient tachypnea of the newborn (TTN) of infected mothers also occurred, admission to the neonatal ICU was supported by non-invasive mechanical ventilation (CPAP). ${ }^{15,20,26,27}$

Universal test for SARS-CoV-2 and evaluation of vertical transmission: Then, in another study conducted between March 22 and April 4, 2020, a total of 215 pregnant women who gave birth to newborns in the Hospital Allen Presbyterian from New York and Columbia University Irving Medical Center, New York-USA All women were screened on admission for Covid-19 symptoms. 1.9\% (CI 95\% 0.5-4.7) of the pregnant women had fever or other symptoms of Covid-19 at admission, and tested positive for SARS-CoV-2, while 98.1\% (CI 99 \% 95.3-99.5) were afebrile upon admission, asymptomatic. Nasopharyngeal swabs were performed at 99.5\% (95\% CI 97.4-100) of asymptomatic pregnant women, of which 13.8\% (95\% CI 9.5-19.2) were positive for SARS-CoV-2, and 10.3\% (CI 95\% 2.227.4) of the latter, fever developed postpartum before discharge (presymptomatic) (Figure 5).

It should be noted that a pregnant patient with a swab that was

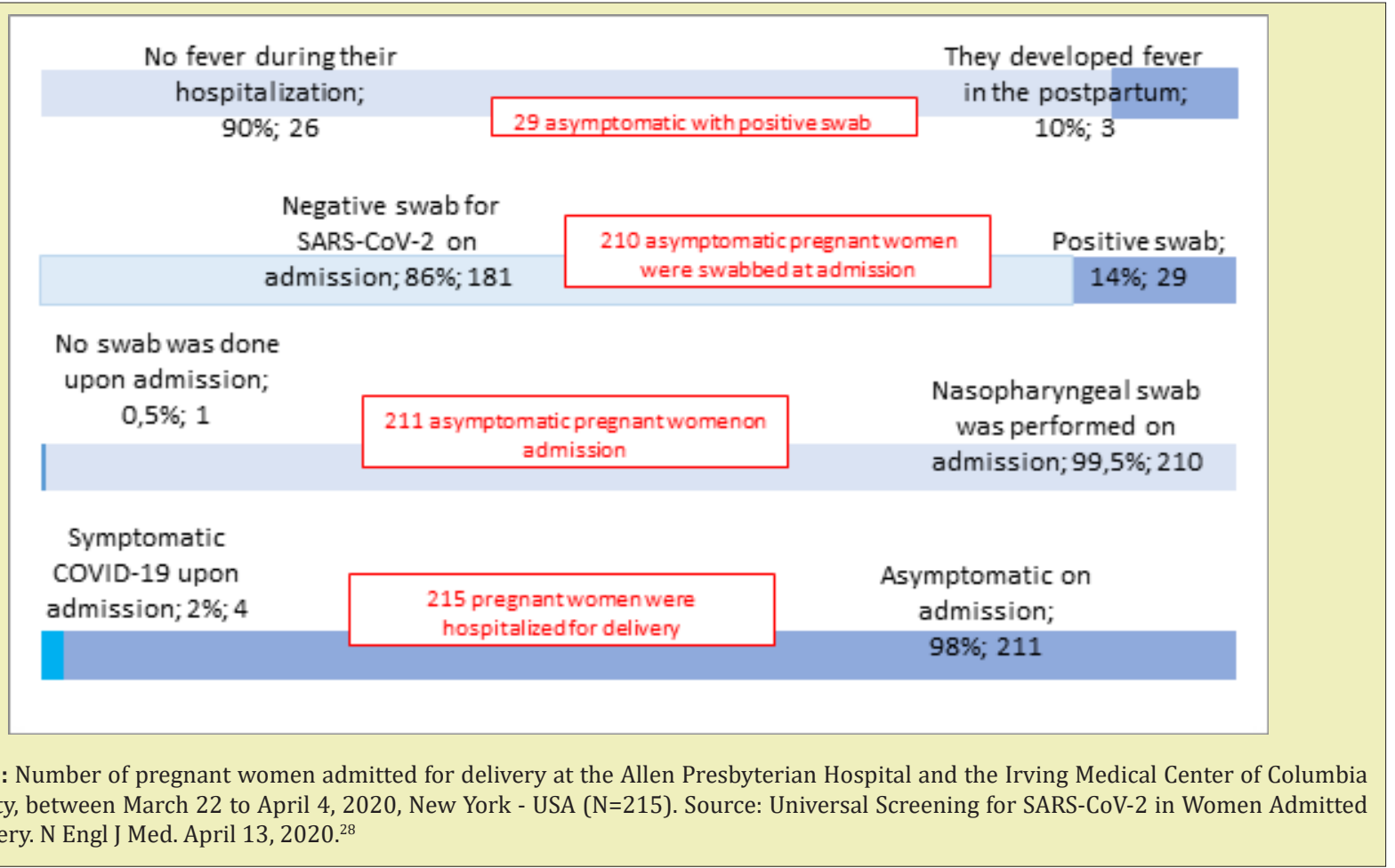

negative for SARS-CoV-2 on admission became postpartum symptomatic; the SARS-CoV-2 test is repeated three days later, resulting in a positive test. ${ }^{28}$ In this way, it is assumed that a group of pregnant women with Covid-19 were asymptomatic before and during delivery since they tested negative for SARS-CoV-2 before delivery, but after delivery they presented symptoms and tested positive for SARS- CoV-2 (presymptomatic), and on the other hand, SARS-
CoV-2 infection has been documented in patients who never develop symptoms (asymptomatic), ${ }^{5,16,28}$ which indicates possible high rates of asymptomatic presentation in this population. This is likely due to the universal detection strategy for Covid-19 in pregnancy and lower thresholds for testing than in cases outside of pregnancy. ${ }^{15}$ The true prevalence of infection may not be reported due to the false negative results of the rt-PCR tests to detect SARS-CoV-2, 
hence the need to focus a universal rt-PCR test on pregnant women to determine the status of Covid-19, that is, that every pregnant woman undergoes the test to rule out SARS-CoV-2 before delivery, and thus points to hospital isolation practices, bed assignments and due treatment to protect mothers, with proper neonatal care and guide the use of personal protective equipment for health personnel during these difficult times. But that, unfortunately, there is insufficient evidence for screening. ${ }^{28,29}$ The sensitivity of the current rt-PCR diagnostic test (to date, considered the gold standard) to detect the SARS CoV2 virus is around $71 \%{ }^{14}$ Therefore, the reliability of diagnostic tests needs to be further evaluated. ${ }^{30}$ On the other hand, the ELISA IgM/IgG serology tests for SARS-CoV-2, which according to manufacture and origin ${ }^{29}$ could yield a sensitivity of $93.8 \%$ and a specificity of $95.6 \%$, would be appropriate for the control evolution of the cases corroborating with the symptoms of the patients, but not for the diagnosis, precisely.,31

For SARS-CoV-2 screening to rule out vertical transmission, amniotic fluid samples from pregnant patients with Covid-19 were obtained by direct syringe aspiration at the time of neonate birth. ${ }^{19}$ Umbilical cord blood, some placentas, genital fluid, ${ }^{17}$ and neonatal oropharyngeal and nasopharyngeal swab samples were collected immediately after delivery in the operating room. Additionally, breast milk samples were collected from colostrum. All the samples analyzed were negative for the virus and none of the neonates had postpartum complications such as Covid-19 infection. ${ }^{18,26}$ Anyway, the possibility that pregnant women infected by SARS-CoV-2 present intrauterine or transplacental transmission to their fetuses cannot be ruled out, according to some researchers, it is possible that it occurs during the last weeks of pregnancy. ${ }^{19}$ However, large-scale histopathological studies of the placenta have not been performed, as more supporting evidence will be required. It should be noted that there are positions that do not recommend late clamping of the umbilical cord during delivery care. ${ }^{32,33}$ Therefore, it will be necessary to collect additional samples from neonates, including nasopharyngeal and oropharyngeal swabs, placenta, amniotic fluid, neonatal blood, gastric fluid, rectal swabs, among others, to detect possible receptors of the virus. ${ }^{26,33}$

On the other hand, it is concerned that under no pretext the real cases of pregnant women with positive Covid-19 are not reported, within the global statistics, and thus not give rise to the under-registration. Without good data, forecasters would have to rely on guesswork and assumptions. ${ }^{29}$

Management of the pregnant woman with Covid-19 in the health system: At this point in the global pandemic of Covid-19 infection, it is important that the pregnant woman stays in contact with the maternity team that assists her, and continues with the controls Routine prenatal care scheduled while the pregnant woman is well and as instructed by the health professional assisting her, as it may be necessary to reduce the number of prenatal check-ups to be had. ${ }^{10}$ If the pregnant woman has mild symptoms of Covid-19, to mention, cough, fever, sore throat, headache, ageusia and anosmia, ${ }^{10}$ after evaluation of her seriousness by health personnel, she must remain isolated at home at least 14 days to 21 days, ${ }^{34,35}$ and communicate with the maternity team to receive the necessary guidance and for them to organize the correct place and time for prenatal check-ups, since clinical and obstetric procedures require special preparations for infection control and protective clothing. ${ }^{36}$ Routine prenatal controls are likely to be delayed until isolation ends, taking into account that below 24 weeks of pregnancy, fetal viability will be checked and the same procedure will be followed as for other Covid-19 patients; and for over 24 weeks, fetal well-being will be checked and the patient will remain under observation until the result of the following tests: X-ray and chest CT, which will not be delayed due to the need to perform it; fetal ultrasound based on the weeks of pregnancy, the severity of the maternal clinical picture and the need to monitor fetal well-being; and if possible, a cardiotocographic record for fetal well-being controls appropriate to the weeks of pregnancy, and based on fetal growth and the mother's condition. $^{37}$

Pregnant women with more severe symptoms due to Covid-19 should be treated by a multidisciplinary team at the hospital level, which only cares for patients who meet these strict classification criteria and does not care for other low-risk patients. ${ }^{7,38}$ In the most serious cases, when the possibility of sepsis or organ failure is detected, the patient should be transferred to an ICU.7,9,15,16 The experimental use of unauthorized drugs outside the scope of a study, for example antivirals, should be guided by an individual analysis of risks and benefits based on the possible benefits to the mother and the safety of the fetus, and should be carried out after consulting a doctor. obstetrician and an ethics committee. ${ }^{13,38}$ Symptomatic treatments should be administered to pregnant women with suspected or confirmed SARS-CoV-2 infection, adjusting them to the physiology of pregnancy. ${ }^{10}$

Since pregnancy is a hypercoagulable and pre-inflammatory state, and emerging evidence suggests that hospitalized patients with Covid-19 are also hypercoagulable, ${ }^{25}$ it follows that infection with Covid-19 is probably associated with an increased risk of maternal venous thromboembolism (VTE). ${ }^{39}$ Reduced mobility due to self-isolation at home or hospital admission would further increase the risk. ${ }^{10,40}$ The researchers assure that it will be the clinicians who will have to choose the moment of delivery, taking into account the week of gestation, maternal and fetal conditions and the delivery itself. Whenever possible, they point out, "vaginal delivery should be favored through induction, with a possible instrumental delivery to avoid maternal exhaustion and unnecessary surgical complications in an already ill patient." Rather, "septic shock, acute organ failure, or fetal distress should lead to emergency cesarean delivery (or termination, if legal, of fetal viability)". ${ }^{9}$ Negative pressure isolation wards for safe delivery should be established for the act. ${ }^{38}$ Decisions about emergency delivery and termination of pregnancy are difficult and based on many factors: gestational age, mother's condition and the stability of the fetus. Consultation with specialists in obstetrics, neonatology, and intensive care (depending on the mother's condition) is essential. ${ }^{41}$ Pregnant women should adopt the same preventive measures as other adults in order to avoid contagion; For example, avoiding direct contact with people who are 
coughing or sneezing, wearing masks and maintaining physical distance from person to person, washing hands with soap and water or alcohol-based solutions frequently, covering mouth and mouth nose with a tissue or elbow when coughing and sneezing, and cooking food thoroughly. ${ }^{42}$

Breastfeeding and risk of infection of the newborn in the context of Covid-19: Breastfeeding has a protective effect against infectious diseases with the direct transfer of antibodies and is the best source of nutrition for babies. ${ }^{5}$ However, doubts arise about what should be done by those nursing mothers who may be infected with SARS-CoV-2. It has been found that it is not transmitted through breast milk. ${ }^{2}$ However, newborns of mothers with suspected or confirmed Covid-19 infection should be isolated for at least 14 days or until the virus clears. ${ }^{36}$ During this time, a group of experts do not recommend direct breastfeeding. The mother should be encouraged to express her milk. Then, when feeding the newborn, use a clean spoon or container, wear a mask, wash hands before and after being in contact with the child, and clean or disinfect contaminated surfaces. ${ }^{11}$ Of course, This will affect early attachment and the establishment of lactation, causing additional stress, anxious or depression for mothers in the postpartum period due to isolation measures. ${ }^{29,32,38}$

The risk of contagion of the virus from the mother to the newborn occurs mainly through close person-to-person contact, through oropharyngeal drops of saliva (between caregivers, relatives and family visitors), ${ }^{43}$ infections acquired in hospital and exposure to sources of infection in public places, ${ }^{17}$ or fecal-oral, ${ }^{44}$ as in the general population. Emphasize the appropriate and maximum care of the management of the uninfected newborn during breastfeeding and other care received, since the case of a full-term newborn, the son of a mother with Covid-19 and born by cesarean section, was reported positive at 30 hours of life in an oropharyngeal swab sample, the baby being asymptomatic. ${ }^{45}$ If the newborn presents fever, lethargy, irritability, poor diet or any other symptom of concern, medical attention should be sought immediately. ${ }^{10}$

\section{Conclusion}

Limitations of the study: It is important to highlight that these findings do not cover all pregnant women, since they have been carried out on a very limited number of cases. For example, it has not yet been possible to study the behavior of exposure to SARS-CoV-2 infection during the first and second trimesters of pregnancy and their follow-up, since no evidence has suggested an association between Covid- 19 and congenital defects.

Context and reality: Based on the contingency due to Covid-19, it is necessary to strengthen telemedicine in our environment, but it still has to overcome difficulties of access to the community, especially rural communities, with low income and with limitations sociocultural, in addition to the lack of specialized biomedical equipment to perform the diagnosis and to have trained personnel to adequately approach the users with professional empathy, human sensitivity and request for service in this case. ${ }^{46}$ Health care
WhatsApp groups of pregnant women have been created, as a follow-up and scheduling mechanism for prenatal controls, either at the health service or at the pregnant woman's home level as the case requires, establishing communication with the more complex health services for the specialized care of pregnant women or for the realization of auxiliary studies (laboratory, ultrasound). ${ }^{5,10}$

However, health services from the first, second and third level of medium complexity that provide multipurpose care to decongest the most specialized hospital services, which include prenatal control and even delivery, are unprotected since they do not have the necessary infrastructure: wards with negative pressure, ultraviolet light, and even personal protective equipment, to address potentially asymptomatic patients, due to the implicit risk of exposure, and at the same time, prevent health workers from transmitting Covid-19. ${ }^{25,29,47}$

Challenges: Efforts should be made to reduce the rate of SARSCoV-2 infection, both in the gestational and perinatal period, and to provide more careful care for pregnant patients. If infection is confirmed during pregnancy, long-term follow-up should be recommended to ensure the well-being of mothers and their fetuses, ${ }^{48}$ SARS-CoV-2 appears to be most contagious at the time of onset of symptoms and Infectivity decreases rapidly thereafter to almost zero after approximately 10 days, in mild, moderate patients and 15 days in severe, critical, and immunosuppressed patients. The longest interval associated with replication competent virus so far is 20 days from symptom onset. ${ }^{5}$

As this global pandemic continues to expand, additional information will be available on the effects of Covid-19 on pregnant women and their babies, such as the emerging variants of SARS$\mathrm{CoV}-2 .{ }^{49}$ In the unfortunate event of mortality resulting from SARS-CoV-2 infection among pregnant women or their neonates, pathological evaluation of the detected tissues along with molecular characterization of the virus would be helpful in determining the pathogenesis of the disease as has been done in many cases of emerging infections. ${ }^{33}$ Then, there have been cases of abortions, maternal and fetal deaths from possible Covid-19, but the available evidence on adverse pregnancy outcomes associated with Covid-19 is limited. ${ }^{33}$ It deserves a separate chapter through extensive monitoring and analysis. The available recommendations on obstetric treatment of SARS-CoV-2 infection in pregnant women should be updated, aiming at the care of the mother-child binomial. Furthermore, it should be remembered that as the development of vaccines for Covid-19 progresses, pregnant women should be considered for inclusion in clinical trials, as well as in the eventual distribution of the vaccine unless the risks outweigh the potential benefits. ${ }^{50}$

Therefore, it is necessary to maintain access to sexual and reproductive health services of the National Health System, to ensure that pregnant women with suspected, probable or confirmed cases of Covid-19, including those who may need to spend time in isolation, have access to qualified care according to a protocol to be followed, including maternal obstetric screening tests, fetal medicine and neonatal care, and mental health and psychosocial support 
with the capacity to treat maternal and neonatal complications. ${ }^{51}$ These proposals may evolve to daily according to the progress of this pandemic and evidence in pregnant women that allow to improve in-depth knowledge on the subject. ${ }^{20}$

\section{Aknowlegdegments}

To the Journal of Pregnancy and Women's Health Care for allowing us to share this information.

\section{Funding}

No financial support.

\section{Conflict of Interest}

No conflict of interest.

\section{References}

1. Zhu N, Zhang D, Wang W, et al. A Novel Coronavirus from Patients with Pneumonia in China, 2019. N Engl J Med. 2020;382(8):727-733.

2. Schwartz DA. An Analysis of 38 Pregnant Women with COVID-19, Their Newborn Infants, and Maternal-Fetal Transmission of SARSCoV-2: Maternal Coronavirus Infections and Pregnancy Outcomes. Arch Pathol Lab Med. 2020;144(7):799-805.

3. WHO. Nuevo coronavirus 2019.

4. JHCHS website. Coronaviruses: SARS, MERS, and COVID-19. 2020.

5. CDC. Coronavirus Disease 2019 (COVID-19).

6. Lüscher TF. Understanding COVID-19: in the end it is the endothelium-what else? Eur Heart J. 2020;41(32):3023-3027.

7. Cheng F, Zhang Y. The Clinical Diagnosis and Treatment for New Coronavirus Pneumonia [Internet]. Singapore: Springer Singapore; 2020.

8. COVID Live Update: 143,027,791 Cases and 3,047,439 Deaths from the Coronavirus-Worldometer. 2021.

9. Favre G, Pomar L, Qi X, et al. Guidelines for pregnant women with suspected SARS-CoV-2 infection. Lancet Infect Dis. 2020;20(6):652-653.

10. RCOG. Coronavirus infection and pregnancy. Royal College of Obstetricians \& amp; Gynaecologists. 2020.

11. UNFPA. Comunicado del UNFPA sobre el nuevo coronavirus (COVID-19) y el embarazo. 2020.

12. WHO-2019-nCoV-immunization_services-2020.1-spa.pdf. 2020.

13. Liu D, Li L, Wu X, et al. Pregnancy and Perinatal Outcomes of Women with Coronavirus Disease (COVID-19) Pneumonia: A Preliminary Analysis. Am J Roentgenol. 2020;215(1):127-132.

14. Fang Y, Zhang H, Xie J, et al. Sensitivity of Chest CT for COVID-19: Comparison to RT-PCR. Radiology. 2020;296(2):E115-E117.

15. Allotey J, Stallings E, Bonet M, et al. Clinical manifestations, risk factors, and maternal and perinatal outcomes of coronavirus disease 2019 in pregnancy: living systematic review and meta-analysis. BMJ. 2020. p. 370.

16. Ellington S. Characteristics of Women of Reproductive Age with Laboratory-Confirmed SARS-CoV-2 Infection by Pregnancy Status-United States, January 22-June 7, 2020. MMWR Morb Mortal Wkly Rep. 2020;69(25):769-775.

17. Zhu $\mathrm{H}$, Wang $\mathrm{L}$, Fang $\mathrm{C}$, et al. Clinical analysis of 10 neonates born to mothers with 2019-nCoV pneumonia. Transl Pediatr. 2020;9(1):5160.
18. Chen H, Guo J, Wang C, et al. Clinical characteristics and intrauterine vertical transmission potential of COVID-19 infection in nine pregnant women: a retrospective review of medical records. The Lancet. 2020;395(10226):809-915.

19. Vivanti AJ, Vauloup Fellous C, Prevot S, et al. Transplacental transmission of SARS-CoV-2 infection. Nat Commun. 2020;11(1):3572.

20. Peyronnet V, Sibiude J, Deruelle P, et al. Infection par le SARS-CoV-2 chez les femmes enceintes. État des connaissances et proposition de prise en charge. CNGOF. Gynécologie Obstétrique Fertil Sénologie. 2020;48(5):436-443.

21. Dubey P, Reddy SY, Manuel S, et al. Maternal and neonatal characteristics and outcomes among COVID-19 infected women: An updated systematic review and meta-analysis. Eur J Obstet Gynecol Reprod Biol. 2020;252:490-501.

22. Charles J. Kahi MD. Gastrointestinal Aspects of COVID-19. NEJM J Watch. 2020.

23. Backer JA, Klinkenberg D, Wallinga J. Incubation period of 2019 novel coronavirus (2019-nCoV) infections among travellers from Wuhan, China, 20-28 January 2020. Eurosurveillance. 2020;25(5).

24. Giacomelli A, Pezzati L, Conti F, et al. Self-reported Olfactory and Taste Disorders in Patients with Severe Acute Respiratory Coronavirus 2 Infection: A Cross-sectional Study. Clin Infect Dis. 2020;71(15):889890 .

25. Berlin DA, Gulick RM, Martinez FJ. Severe Covid-19 [Internet]. New England Journal of Medicine. 2020.

26. Chen Y, Peng $\mathrm{H}$, Wang $\mathrm{L}$, et al. Infants Born to Mothers with a New Coronavirus (COVID-19). Front Pediatr. 2020;8:104.

27. Knight M, Bunch K, Vousden N, et al. Characteristics and outcomes of pregnant women admitted to hospital with confirmed SARS-CoV-2 infection in UK: national population based cohort study. BMJ. 2020;369.

28. Sutton D, Fuchs K, D'Alton M, et al. Universal Screening for SARS-CoV-2 in Women Admitted for Delivery. N Engl J Med. 2020;382(22):21632164

29. Schneider EC. Failing the Test-The Tragic Data Gap Undermining the U.S. Pandemic Response. N Engl J Med. 2020;383(4):299-302.

30. Joung J, Ladha A, Saito M, et al. Detection of SARS-CoV-2 with SHERLOCK One-Pot Testing. N Engl J Med. 2020. p. 4.

31. JHCHS website administrator. Global Progress on COVID-19 Serology-Based Testing. Johns Hopkins Center for Health Security. 2020.

32. Wang L, Shi Y, Xiao T, et al. Chinese expert consensus on the perinatal and neonatal management for the prevention and control of the 2019 novel coronavirus infection (First edition). Ann Transl Med. 2020;8(3):47.

33. Kirtsman M, Diambomba Y, Poutanen SM, et al. Probable congenital SARS-CoV-2 infection in a neonate born to a woman with active SARSCoV-2 infection. CMAJ. 2020;192(24):E647-E650.

34. Linton NM, Kobayashi T, Yang Y, et al. Incubation Period and Other Epidemiological Characteristics of 2019 Novel Coronavirus Infections with Right Truncation: A Statistical Analysis of Publicly Available Case Data. J Clin Med. 2020;9(2):538.

35. Remuzzi G, Perico N, Suter F. A recurrent question from a primary care physician: How should I treat my COVID-19 patients at home? 2020.

36. Qiao J. What are the risks of COVID-19 infection in pregnant women? The Lancet. 2020;395(10226):760-762.

37. Martinez Portilla RJ, Goncé A, Hawkins Villarreal A, et al. A Spanish-translated clinical algorithm for management of suspected SARSCoV-2 infection in pregnant women. Lancet Infect Dis. 2020. 
38. Chua MSQ, Lee JCS, Sulaiman S, et al. From the frontline of COVID-19 - How prepared are we as obstetricians: a commentary. BJOG. Int J Obstet Gynaecol. 2020;127(7):786-788.

39. SETH. Recomendaciones sobre profilaxis de enfermedad tromboembólica (ETV) en el embarazo y puerperio durante la pandemia COVID-19 [Internet]. COVID-19 - Sociedad Española de Trombosis y Hemostasia. 2020.

40. Beverley Hunt B, Retter A, McClintock C. et al. The Thrombosis Charity wishes to increase awareness of thrombosis among the public and health professionals and to raise research funds to improve patient care. Helping people who suffer from VTE, DVT, PE and clots. 2020.

41. Lu Z, Yan J, Min W, et al. Analysis of the pregnancy outcomes in pregnant women with COVID-19 in Hubei Province. Chin J Obstet Gynecol. 2020;55(00):E009-E009.

42. WHO. \#HealthyAtHome. 2020.

43. Coronado Munoz A, Nawaratne U, McMann D, et al. Late-Onset Neonatal Sepsis in a Patient with Covid-19. N Engl J Med. 2020;382(19):e49.

44. Yongjian $\mathrm{W}$, Cheng G, Lantian T, et al. Prolonged presence of SARSCoV-2 viral RNA in faecal samples. The Lancet Gastroenterology \&Hepatology. 2020;5(5):434-435.
45. Lu Q, Shi Y. Coronavirus disease (COVID-19) and neonate: What neonatologist need to know. J Med Virol. 2020;1-4.

46. Rivas R, Galván P. Telemedicina en el Paraguay: Aportes del Instituto de Investigaciones en Ciencias de la Salud, Universidad Nacional de Asunción (IICS-UNA). MemInst Investig En Cienc Salud. 2018;16(3):66-72.

47. McCullough PA, Kelly RJ, Ruocco G, et al. Pathophysiological Basis and Rationale for Early Outpatient Treatment of SARS-CoV-2 (COVID-19) Infection. Am J Med. 2020;0(0).

48. Favre G, Pomar L, Musso D, et al. 2019-nCoV epidemic: what about pregnancies?. The Lancet. 2020;395(10224):e40.

49. CDC. El COVID-19 y su salud. Centers for Disease Control and Prevention. 2020

50. Picazo JJ. Vacuna frente al COVID-19. Soc Esp Quimioter Infecc Vacunas. 2020. p. 12.

51. UNFPA. La Salud y los Derechos Sexuales y Reproductivos, la Salud Materna y Neonatal y la COVID-19. 2020 